Deutsche Gesellschaft für Hämatologie und Onkologie e.V. Geschäftsführender Vorsitzender Prof. Dr. Gerhard Ehninger V.i.S.d.P. Mirjam Renz

Alexanderplatz 1, 10178 Berlin, Germany

Tel. +4930 2787 6089-0, Fax -18

renz@dgho.de

www.dgho.de

\title{
DGHO plädiert für wissenschaftlich fundierte Nutzenbewertung neuer Krebsmedikamente
}

Um den tatsächlichen therapeutischen Nutzen neuer Krebsmedikamente schnellstmöglich festzustellen, müssen industrieunabhängige Daten sowie die Expertise der Fachmedizin stärker berücksichtigt werden. Dies fordert die Deutsche Gesellschaft für Hämatologie und Onkologie e.V. (DGHO) anlässlich der Jahrestagung 2010 der deutschen, österreichischen und schweizerischen Gesellschaften für Hämatologie und Onkologie. Vom 1. bis 5. Oktober 2010 trafen sich im ICC / Messe Berlin mehr als 4500 Experten, um über den aktuellen Forschungsstand in Diagnose und Therapie von Blut- und Krebserkrankungen zu diskutieren. Die Jahrestagung ist eine der wichtigsten Plattformen für den wissenschaftlichen Austausch und gesundheitspolitischen Dialog auf dem Gebiet der Hämatologie und Onkologie.

Die diesjährige Jahrestagung stand unter dem Motto «Der Weg zur personalisierten Krebsmedizin». Die Entdeckung molekularer Mechanismen der Krankheitsentstehung hat in den letzten Jahren zu beachtlichen Fortschritten in der Therapie von Blut- und Krebserkrankungen geführt. Krankheiten wie Leukämie oder Brustkrebs stellen sich immer mehr als heterogene Erkrankungen mit individuellen biologischen und genetischen Eigenschaften dar. Basierend auf diesen Erkenntnissen forscht die Biomedizin an zielgerichteten Therapiestrategien, die das individuelle Ansprechen verbessern und unnötige Nebenwirkungen vermeiden sollen. «Als Fachärzte und Fachgesellschaften müssen wir neue Therapieoptionen prüfen, kritisch bewerten oder wenn nötig auch verwerfen, um neue Standardtherapien festlegen zu können», erläutert der Kongresspräsident Prof. Eckhard Thiel, Direktor der Medizinischen Klinik mit Schwerpunkt Hämatologie und Onkologie der Charité Campus Benjamin Franklin.
Industrieunabhängige Daten für die Nutzenbewertung neuer Medikamente

Zielgerichtete medikamentöse Therapien sind teuer, und ihr tatsächlicher therapeutischer Nutzen zeigt sich in der Onkologie oft erst nach Jahren. Daher begrüßt die DGHO grundsätzlich das geplante Arzneimittelneuordnungsgesetz (AMNOG), das unter anderem bei der Bewertung des Nutzens neuer Medikamente mehr Transparenz schaffen soll. «Allerdings ist nicht nachvollziehbar, warum der Gesetzgeber das fachmedizinische Wissen und industrieunabhängige Daten nicht stärker berücksichtigt», kritisiert Prof. Gerhard Ehninger, Geschäftsführender Vorsitzender der DGHO. «Die derzeit geplanten Regelungen zur Nutzenbewertung neu zugelassener Medikamente führen in dieser Form nicht zu einer Weiterentwicklung der Onkologie», ergänzt Ehninger.

Die DGHO hält eine Nachbesserung für dringend geboten. Wissenschaftlich-medizinische Fachgesellschaften müssen laut DGHO ein Anhörungs- und Beteiligungsrecht bei der Erstellung der Nutzen- bzw. der Kosten-Nutzen-Bewertung erhalten. Bei der Festlegung der Methoden und Kriterien für den Prüfauftrag an das Institut für Qualität und Wirtschaftlichkeit im Gesundheitswesen (IQWiG) sollten Fachgesellschaften einbezogen werden. Außerdem müsse der Gemeinsame Bundesausschuss Versorgungsstudien nicht nur mit pharmazeutischen Unternehmen, sondern auch mit Kompetenznetzen, Studiengruppen und Fachgesellschaften vereinbaren können.

\section{KARGER}

Fax +497614520714

Information@Karger.de

www.karger.com (c) 2010 S. Karger GmbH, Freiburg

Accessible online at:

www.karger.com/onk 
Defizite bei den Zulassungsstudien neuer Krebsmedikamente

In den letzten Jahren wurden immer mehr, häufig sehr teure onkologische Arzneimittel neu zugelassen. «Schon zum Zeitpunkt der Zulassung gibt es ein erhebliches Erkenntnisdefizit bezüglich der Wirksamkeit und Sicherheit neuer Wirkstoffe», erklärt Prof. Wolf-Dieter Ludwig, Vorsitzender der Arzneimittelkommission der deutschen Ärzteschaft. «Infolge ökonomischer Interessen und Marketingaspekten werden patientenrelevante primäre Endpunkte wie Gesamtüberleben und Lebensqualität in Zulassungsstudien häufig nur unzureichend berücksichtigt», so Ludwig weiter. Um den Nutzen bzw. das Risiko der neuen Arzneimittel ausreichend zu erfassen, schlägt Ludwig vor, dass sich Experten unmittelbar nach $\mathrm{Zu}$ lassung zusammensetzen, um neue Wirkstoffe hinsichtlich ihres therapeutischen (Zusatz-)Nutzens zu bewerten und offene Fragen für unabhängige, versorgungsrelevante PhaseIV-Studien festzulegen.

\section{"Onkopedia" - Therapieempfehlungen \\ für Ärzte und Patienten}

«Aufgrund der Vielzahl neuer Studien in der Onkologie brauchen Ärzte ein verlässliches Instrument für die individuelle Therapieentscheidung, das den aktuellen Stand des Wissens komprimiert und übersichtlich darstellt», erläutert Prof. Bernhard Wörmann, Facharzt für Hämatologie und Internistische Onkologie und DGHO-Koordinator für Leitlinien. Die DGHO hat deshalb die onkologische Wissensdatenbank «Onkopedia» im Internet angelegt. Seither bearbeiten und aktualisieren Experten der DGHO regelmäßig Therapieempfehlungen zu den häufigsten Erkrankungen im Bereich Hämatologie und Onkologie.

In Kürze stehen unter «Mein Onkopedia» für Krebspatienten, Angehörige und Interessierte auch die ersten 15 Patientenleitlinien zur Verfügung. Sie basieren auf den aktuellen DGHO-Therapieempfehlungen und sind teilweise in Zusammenarbeit mit Selbsthilfeorganisationen verfasst worden. Fachbegriffe werden in einem getrennten Glossar erklärt.

\section{Preisverleihungen im Rahmen des wichtigsten Kongresses der deutschsprachigen Hämatologen und Onkologen}

Im Rahmen der Jahrestagung der deutschsprachigen Gesellschaften für Hämatologie und Onkologie aus Deutschland, Österreich und der Schweiz ehren die Fachgesellschaften auch ihren wissenschaftlichen Nachwuchs in der Krebsmedizin. Zwei der Preise gehen in diesem Jahr an junge Forscher aus Berlin.

\section{DGHO Doktoranden-Förderpreis 2010}

Für seine Arbeit zur Signaltransduktion in blutbildenden Zellen (Ubiquitination and degradation of the thrombopoietin receptor c-Mpl.) erhält Sebastian-Jonas Saur aus Freiburg in diesem Jahr den Doktoranden-Förderpreis der DGHO. Saur konnte sich damit gegen zahlreiche Nachwuchswissenschaftler durchsetzten, die sich um diesen Preis beworben hatten. Seine Arbeit hat Auswirkungen auf unser Verständnis der Differenzierung von Stammzellen. Das Preisgeld beträgt 2500 Euro.

\section{DGHO Forschungs- und Innovationspreis 2010}

Dr. med. Claudia Scholl erhält den von der Firma Pfizer (ehemals Wyeth) gestifteten und mit 7500 Euro dotierten Preis für ihre translationale Forschung. Der Preis wird an Nachwuchswissenschaftler vergeben, die sich vor allem mit Signaltansduktion bei Krebserkrankungen befassen. In ihrer Arbeit (Synthetic lethal interaction between oncogenic KRAS dependency and STK33 suppression in human cancer cells) konnte Scholl neue Erkenntnisse zu den Signalwirkungen in menschlichen Krebszellen vorlegen, die Einfluss auf zukünftige zielgerichtete Therapien vom Tumorerkrankungen haben.

\section{Vincenz Cerny Preis 2010}

PD Dr. med. Gunnar Folprecht aus Dresden erhält in diesem Jahr den mit 7500 Euro dotierten Vincenz Cerny Preis für herausragende Forschung im Bereich der Onkologie. Folprecht hat in einer multizentrischen klinischen Studie neue Behandlungsmöglichkeiten bei Patienten mit Lebermetastasen eines Dickdarmkarzinoms durch die Kombination von Chemotherapie und nachfolgender Operation aufgezeigt (Tumour response and secondary resectability of colorectal liver metastases following neoadjuvant chemotherapy with cetuximab: the CELIM randomised phase 2 trial). 
Sie vielen Jahren beschäftigte sich Dr. Stephan Mathas aus Berlin mit der Pathogenese und der Entwicklung möglicher neuer Therapieansätze des Hodgkin-Lymphoms sowie des anaplastisch großzelligen Lymphoms. Dabei liegt der Schwerpunkt der Arbeiten vor allem in der Analyse der Mechanismen und Konsequenzen der zellulären Dedifferenzierung bösartiger hämatopoetischer Zellen. Für diese Arbeit wird Mathas im Rahmen der Jahrestagung 2010 mit dem Artur Pappenheim Preis ausgezeichnet, der mit 7500 Euro dotiert ist.
Ein weiterer Preis wird in diesem Jahr einmalig verliehen und geht ebenfalls nach Berlin. PD Dr. med. Claudia D. Baldus aus Berlin erhält für ihre Leukämie-Forschung (Prognostische Bedeutung der molekularen Marker BAALC und ERG bei akuten Leukämien) den Preis der Alfred und Angelika Gutermuth-Stiftung. Dotiert ist die Auszeichnung mit 10000 Euro. Die Medizinerin hat in ihren Arbeiten molekulare Marker identifiziert, die unabhängig vom Leukämiesubtyp erwachsene Patienten mit einem hohen Rezidivrisiko charakterisieren. Die 1998 errichtete Gutermuth-Stiftung (www.gutermuth-stiftung.de) fördert Wissenschaft und Forschung auf dem Gebiet der bösartigen Erkrankungen des blutbildenden Systems. Sie ist insbesondere in Berlin, Frankfurt am Main und Mannheim aktiv. 